\title{
Cloning, Eukaryotic Expression Vector Construction and Tissue Expression Analysis of Mir-133b in Pigs
}

\author{
Lili Yang, Qiongwen Zhang, Xiaotong Gao, Sanbao Zhang, Xiaoxiao Liu, Ting Chen, Qinyang \\ Jiang and Mingsheng Jiang* \\ College of animal science and technology, Guangxi University, China
}

*Corresponding author: Mingsheng Jiang, College of animal science and technology, Guangxi University, Nanning, Guangxi, 530000, China

ARTICLE INFO

Received: 宒 July 16, 2021

Published: 慧 August 10, 2021

Citation: Lili Yang, Qiongwen Zhang, Xiaotong Gao, Sanbao Zhang, Mingsheng Jiang, et al., Cloning, Eukaryotic Expression Vector Construction and Tissue Expression Analysis of Mir-133b in Pigs. Biomed J Sci \& Tech Res 38(1)-2021. BJSTR. MS.ID.006089.

Keywords: Pigs; miR-133b; Eukaryotic Expression Vector; Tissue Expression Analysis

\begin{abstract}
This experiment aims to construct the eukaryotic expression vector of pig pEGFP-N1miR-133b and detect its expression in pig tissues. Using DLY (Duroc $\times$ Landrace $\times$ Yorkshire) growing-finishing pig genomic DNA as a template, the miR-133b precursor and part of its flanking sequence were cloned by PCR technology and inserted into a eukaryotic expression vector using conventional molecular cloning technology to obtain pEGFPN1-miR-133b. The recombinant plasmid was transfected into 3T3-L1 cells by liposome method and observed the fluorescence. Real-time qPCR was used to detect the expression of miR-133b in heart, liver, spleen, lung, longissimus dorsi, subcutaneous fat, and abdominal fat of pigs. The results showed that miR-133b was successfully amplified. 3T3-L1 cells were transfected with pEGFP-N1-miR-133b eukaryotic expression vector and pEGFP-N1 empty plasmid respectively for 48 hours, and the cells had green under a fluorescent inverted microscope. Fluorescence expression proves that the recombinant plasmid can be expressed in 3T3 cells. Using qPCR technology to detect the expression of miR-133b in 3T3 cells, the relative expression of the pEGFP-N1-miR-133b eukaryotic expression vector transfection group was extremely significantly higher than that of the empty group. The qPCR results showed that miR-133b was expressed highest in the longissimus dorsi muscle, followed by the heart, and the lowest expression in fat. In this study, the eukaryotic expression vector of pig miR-133b was successfully constructed, which provided a reference for studying the mechanism of miR-133b on pig muscle development.
\end{abstract}

\section{Introduction}

Non-coding RNAs refer to RNAs that cannot encode proteins, among which microRNAs (miRNAs) are a class of small singlestranded RNAs with a length between 20nT-25nt. As an endogenous and highly conserved ncRNA, miRNAs play a role in muscle development and fat metabolism of animals [1], disease regulation [2] mainly playing a role in post-transcriptional regulation. In recent years, there have been more studies on miRNAs in domestic animals, especially domestic pigs, which are economic animals. Studies have reported that there are 10 significantly different miRNAs in muscle tissue between Laiwu pigs and white pigs [3]. MiR-204 regulates piglets' resistance to Clostridium perfringens type C infection [4]; In the study of Meishan pigs, it was found that miR-208b can affect the transformation of different muscle fiber types by targeting the inhibition of Mettl8 [5].

Initially, miR-133b was thought to be specifically expressed in muscle with a tissue-specific expression pattern, and highly expressed in the heart and skeletal muscle, and playing a key regulatory role in muscle development and remodeling [6]. It has been reported that miR-133b can be used as an indicator for early diagnosis of diabetic nephropathy [7] and can regulate the proliferation process of gastric cancer cells [8]. MiR-133b-siRT1$\beta$-catenin axis can be involved in sporadic gliomas [9]. The function 
and target genes of miR-133b have been extensively studied, and the study on pigs is worth exploring. In this paper, the tissue expression profile of miR-133b was drawn and the eukaryotic expression vector pEGFP-N1-miR-133b was constructed, laying a theoretical foundation for further exploring the mechanism of miR$133 \mathrm{~b}$ in pigs.

\section{Materials and Methods \\ The Experimental Materials}

Blood and tissue samples, pEGFP-N1 eukaryotic expression vector and 3T3-L1 cells of Du adult pigs were stored and provided by College of Animal Science and Technology, Guangxi University. Blood DNA extraction kit, plasmid extraction kit, endotoxic plasmid extraction kit, gel recovery kit, miRNA reverse transcription kit, restriction enzymes BamH I and Hind parabola, pMD19-T vector, T4 ligase, Trizol, etc. were purchased from Baori Medical Technology
(Beijing) Co., Ltd.DH5 $\alpha$ competent cells were purchased from Beijing Quansi Gold Biotechnology Co., Ltd. Lip3000 transfection reagent and fetal bovine serum were purchased from Thermo Fisher Technologies. PBS, DMEM and Trypsin were purchased from Beijing Solibol Technology Co., Ltd.

\section{Primer Design and Synthesis}

The precursor sequence and flanking sequence of miR-133b were searched in the NCBI and UCSC websites, and the cloning primers of miRNA (miR-133BF1, R1) were designed using Oligo 7.0, in which the underlined sequence was the enzyme digestion sequence. The upstream of miR-133b specific quantitative primer was the sequence itself, and the downstream was the general primer. U6 was selected as the internal reference gene, and the primer sequences were shown in Table 1. All primers were synthesized by Nanjing Nuvezan Biotechnology Co., Ltd.

Table 1: Primer's information.

\begin{tabular}{|c|c|c|c|}
\hline Genes & Primer sequences $\left(5^{\prime} \rightarrow 3^{\prime}\right)$ & Product length/bp & Application \\
\hline \multirow{2}{*}{$\operatorname{miR}-133 b$} & F1:CGCGGATCCAGTCTGATTCATGCTAATGCC & \multirow{2}{*}{332} & \multirow{2}{*}{ cloning } \\
\hline & R1:CCAAGCTTCAGGTAATTTCAGCCACAGT & & \\
\hline miR-133b & F2:TTTGGTCCCCTTCAACCAGCTAT & 80 & qPCR \\
\hline \multirow{2}{*}{ U6 } & F:TGGAACGCTTCACGAATTTGCG & \multirow{2}{*}{94} & \multirow{2}{*}{ qPCR } \\
\hline & R:GGAACGATACAGAGAAGATTAGC & & \\
\hline
\end{tabular}

\section{Total RNA Extraction and cDNA Synthesis}

Total RNA was extracted from porcine heart, liver, spleen, lung, kidney, dorsal muscle, subcutaneous fat, abdominal fat and other tissues by Trizol method. RNA with OD260nm/280nm value ranging from 1.8-2.0 was reverse transcribed by miRNA and cDNA was synthesized. The 3T3-L1 cells were washed with PBS for three times, then Trizol lysate was added to collect the cells, and the RNA extraction and reverse transcription methods were the same as the tissues.

\section{Cloning of miR-133b}

Blood DNA extraction kit was used to extract DNA from the whole blood of Dugang pigs, and it was used as a template for amplification of miR-133b precursors and part of the flanking sequences. The PCR reaction system was $10 \mu \mathrm{L}$, of which Ex Taq PCR Mix was $5 \mu \mathrm{L}$, both upstream and downstream primers were added $0.5 \mu \mathrm{L}$, DNA template was $1 \mu \mathrm{L}$, and RNase free $\mathrm{H} 20$ was added to the complement to $10 \mu \mathrm{L}$. The PCR amplification conditions were denaturation at $98^{\circ} \mathrm{C}$ for $10 \mathrm{~s}$, annealing at $55^{\circ} \mathrm{C}$ for $30 \mathrm{~s}$, and extension at $72^{\circ} \mathrm{C}$ for $30 \mathrm{~s}$, with a total of 35 cycles. The PCR products were detected by $1.5 \%$ agrose-gel electrophoresis. After the target strips were recovered and purified, they were attached to pMD19-T vector overnight at $4^{\circ} \mathrm{C}$, transformed into DH5 $\alpha$ capable cells and coated on LA plate containing ampicillin. After $12 \mathrm{~h}$, a single positive clone colony was selected for expansion culture, and the bacterial fluid was identified by PCR. The bacterial fluid with the correct band size was sequenced by Shanghai Sangong.

\section{Construction and Identification of the Eukaryotic Expression Vector of n1-miR-133b}

A small amount of plasmid was extracted from the bacterial fluid with correct sequencing results. The pEGFP-N1 vector and pMD19t-miR-133b recombinant plasmid were digested with BamH I and Hind anion, and the bands were detected by 1.5\% AGAR gel electrophoresis and the gel was recovered. After that, the purified target fragment and N1 were ligated overnight with T4 ligase at $4^{\circ} \mathrm{C}$ without load. After the ligation products were transformed, coated, selected and shaken, PCR identification and sequencing were performed on the bacterial fluid. The product with correct sequencing results was expanded to $6 \mathrm{ml}$, and then the endotoxinfree plasmid was extracted to obtain the eukaryotic expression vector of $\mathrm{n} 1-\mathrm{miR}-133 \mathrm{~b}$, which was stored in a refrigerator at $-40^{\circ} \mathrm{C}$.

\section{Verification of Overexpression of miR-133b in 3T3-L1 Cells}

The resuscitated $3 \mathrm{~T} 3$ cells were cultured in $35 \mathrm{~mm}$ culture dishes and inoculated in 6-well cell culture plates when the cells grew to $90 \%$. When the degree of cell confluence reached about $70 \%$, the empty plasmid pEGFP-N1 and the recombinant plasmid n1-miR-133b were transfected into 3T3 cells using LIP3000 transfection reagent, with 3 replicates for each. The expression of green fluorescence was observed under an inverted microscope 48 hours after transfection and photographed. 


\section{Real-Time Quantitative PCR (qPCR)}

The cDNA of different tissues and cells was used as the template, and U6 was used as the internal reference gene for qPCR verification. The reaction system was as follows: TB Green II 5.0 $\mu \mathrm{l}$, upper, lower primers $0.3 \mu \mathrm{l}$ each, cDNA template $2.5 \mu \mathrm{l}$, RNASEfree $\mathrm{H} 201.9 \mu \mathrm{l}$, and each system was repeated with 3 holes. PCR amplification procedure: pre-denaturation at $95^{\circ} \mathrm{C}$ for $30 \mathrm{~s} ; 45$ cycles of amplification were performed at $95^{\circ} \mathrm{C}$ for $5 \mathrm{~s}$ and $60^{\circ} \mathrm{C}$ for $30 \mathrm{~s}$ to read fluorescence signals. At $65^{\circ} \mathrm{C}$, increase by $0.5^{\circ} \mathrm{C}$ to $95^{\circ} \mathrm{C}$ every $5 \mathrm{~s}$, and read the melting curve. The relative expression level of miR-133b was calculated by $2-\Delta \Delta \mathrm{CT}$ method. Data were analyzed by SPSS 9.4 for one-way ANOVA, $\mathrm{P}<0.05$ indicates significant difference, $\mathrm{P}<0.01$ indicates a significant difference.

\section{Results and Analysis}

\section{Expression Analysis of miR-133b Tissue}

The expression levels of miR-133b in heart, liver, spleen, lung, kidney, dorsal muscle, subcutaneous fat and abdominal fat of Duroc pigs were detected by real-time quantitative PCR, and the results were shown in Figure 1. The expression abundance of miR133b was the highest in the muscle tissue, followed by the heart, which was significantly higher than other tissues $(\mathrm{P}<0.01)$. It can be inferred that miR-133b plays an important regulatory role in muscle, which is consistent with the characteristic of tissue-specific expression of miRNA (Figure 2).

Figure 1: Tissue expression profile of miR-133b.

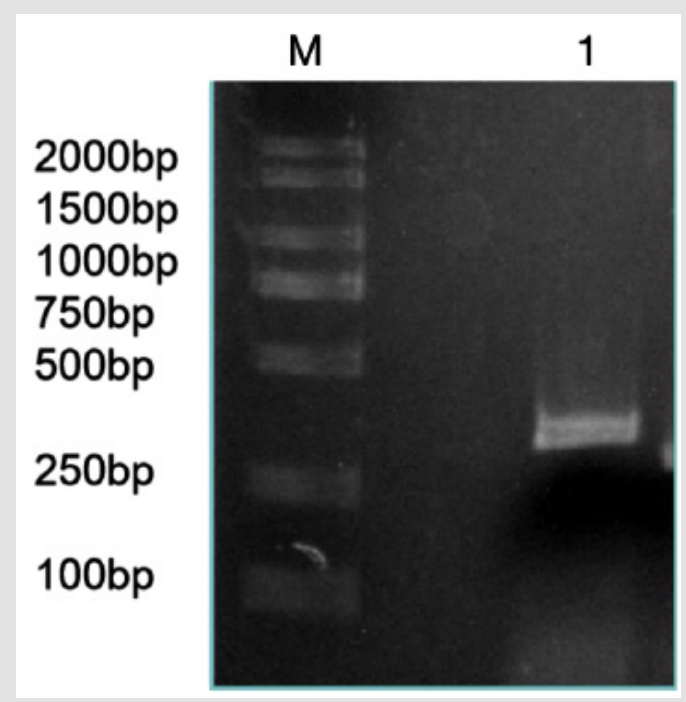

Figure 2: RT-RCR products of miR-133b and its flanking sequence.

Note: M: Marker1; 1: RCR products of miR-133b and its flanking sequence. 


\section{RT-PCR Amplification of miR-133b}

The precursor and part of the flanking sequence of miR-133b were successfully amplified using the DC-grown pig gDNAs as the template. The electrophoresis target band was single and bright, with a size of about $300 \mathrm{bp}$ consistent with the expected fragment (Figure 2). RT-PCR identification of bacterial fluid also showed clear bands of the same size (Figure 3). Sequencing results of Sangon Bioengineering (Shanghai) Co., Ltd showed that miR-133b was successfully amplified with a fragment size of $332 \mathrm{bp}$.

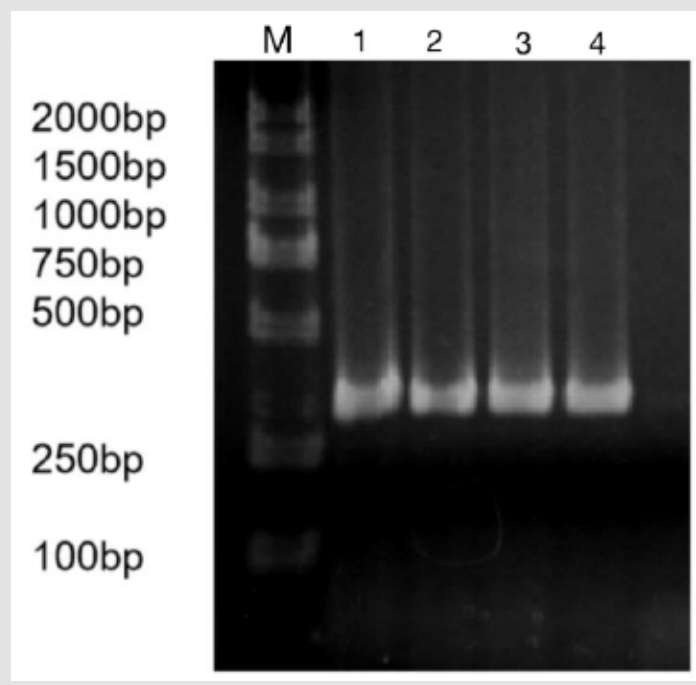

Figure 3: Bacteria liquid PCR products of miR-133b.

Note: M: Marker1; 1 4: Bacteria liquid PCR of miR-133b.

Verification of Recombinant Plasmid pEGFP-N1-miR133b

The constructed eukaryotic expression vector of n1-miR$133 \mathrm{~b}$ was identified by double enzyme digestion, and two clear target bands with correct size and position were obtained by RTPCR amplification (Figure 4). The fragment size of miR-133b was
$268 \mathrm{bp}$, indicating that the vector of $\mathrm{n} 1-\mathrm{miR}-133 \mathrm{~b}$ was successfully constructed. The sequencing results of the recombinant plasmid vector were compared with the sequences provided by the NCBI website, and the results showed that the homology between miR$133 \mathrm{~b}$ of Ductus giant pig and the published seed sequences reached $100 \%$ (Figure 5). 1 is N1 plasmid double digestion, 2 is the result of pMD19T-MI-133B double digestion.

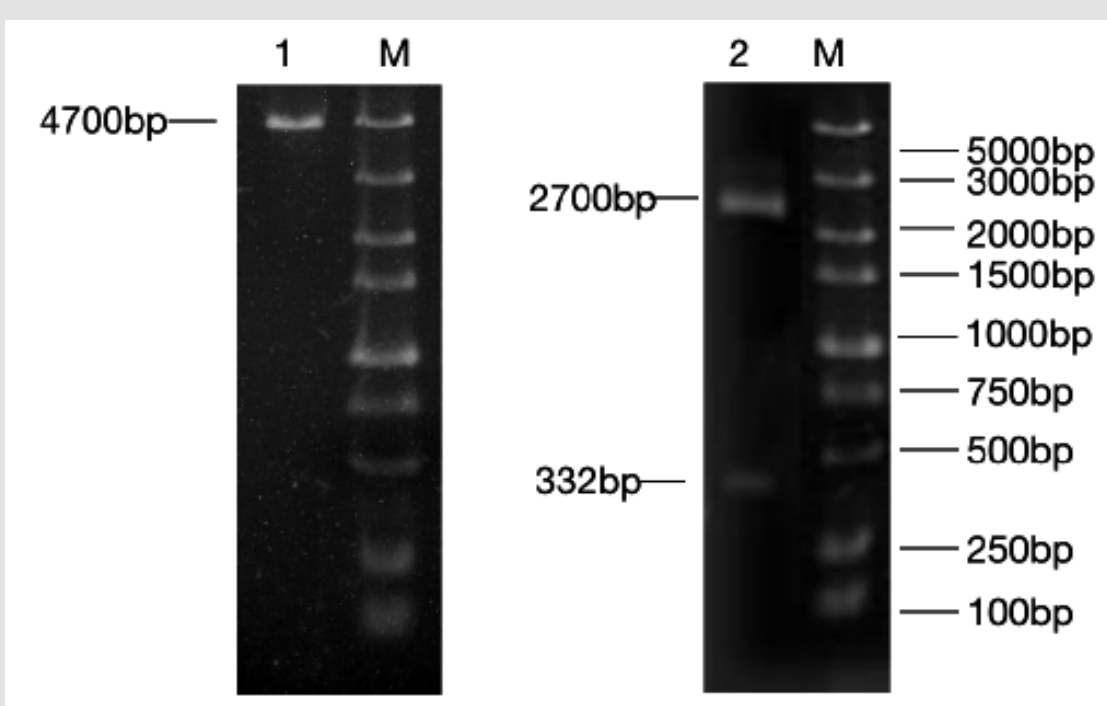

Figure 4: N1,pEGFP-N1-miR-133b recombinant plasmid double digestion and validation.

Note: M: Marker1;1: Double digestion of N1 plasmid 2:PCR products of pEGFP-N1-miR-133b. 


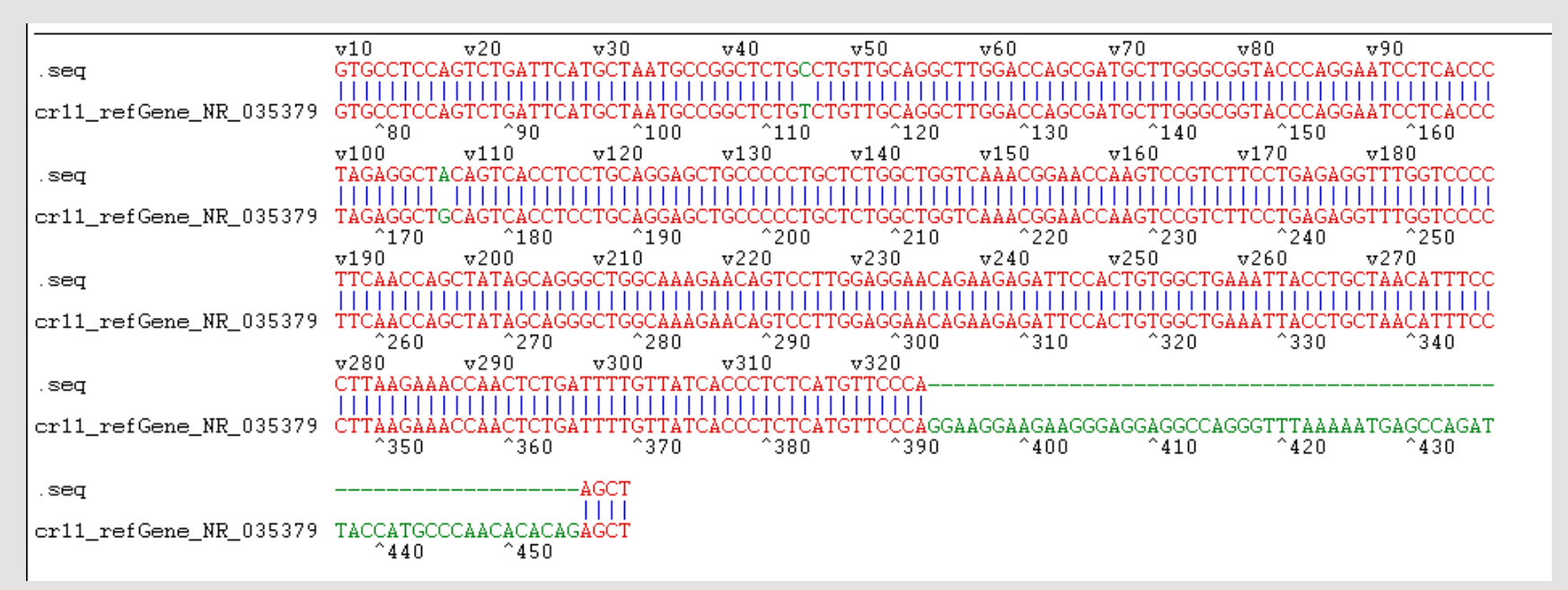

Figure 5: Sequencing identification of pEGFP-N1-miR-133b.

\section{The Recombinant Plasmid pegfp-n1-mir-133b was} Transfected into 3T3 Cells

Pegfp-n1-mir-133b eukaryotic expression vector and pEGFP-N1 empty vector were transfected into 3T3-L1 cells respectively. After 48 hours, green fluorescence expression was observed in 3T3-L1 cells under fluorescence inverted microscope (Figure 6), which proved that the recombinant plasmid could be expressed in $3 \mathrm{~T} 3$ cells. The expression of miR-133b in 3T3 cells was detected by qPCR. The results showed that the relative expression level of pegfp-n1-mir-133b eukaryotic expression vector transfection group was significantly higher than that of empty vector group (P $<0.01$ ) (Figure 7).

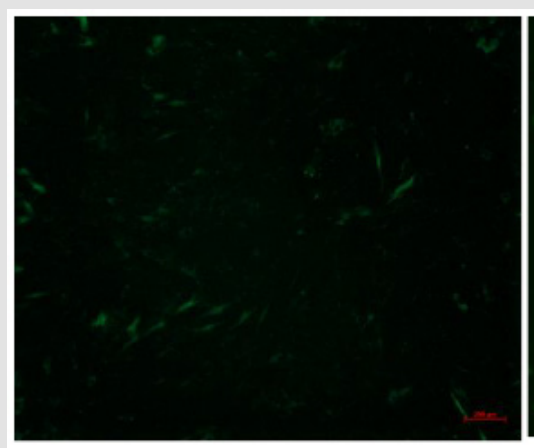

A

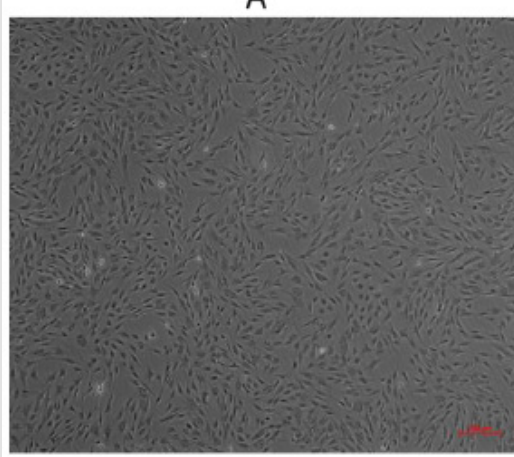

$A^{\prime}$

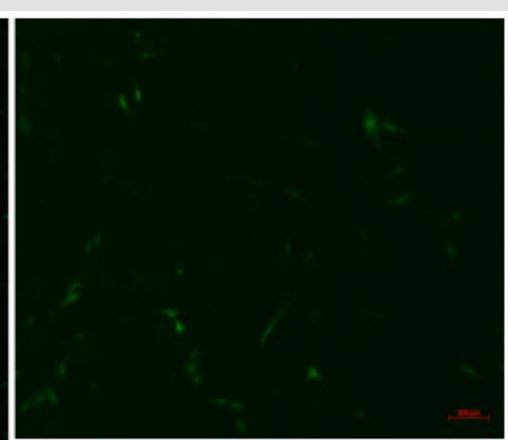

B

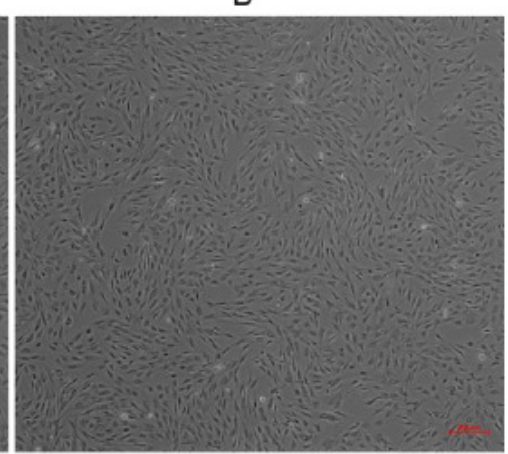

$B^{\prime}$

Figure 6: Green fluorescence results of $3 \mathrm{~T} 3$ cells transfected with recombinant plasmid pEGFP-N1-miR-133b.

Note: A, B were fluorescence of transfection withpEGFP-N1-miR-133b, pEGFP-N1 respectively; A', B'were transfection with EGFP-N1-miR-133b, pEGFP-N1 under under the common light respectively. 


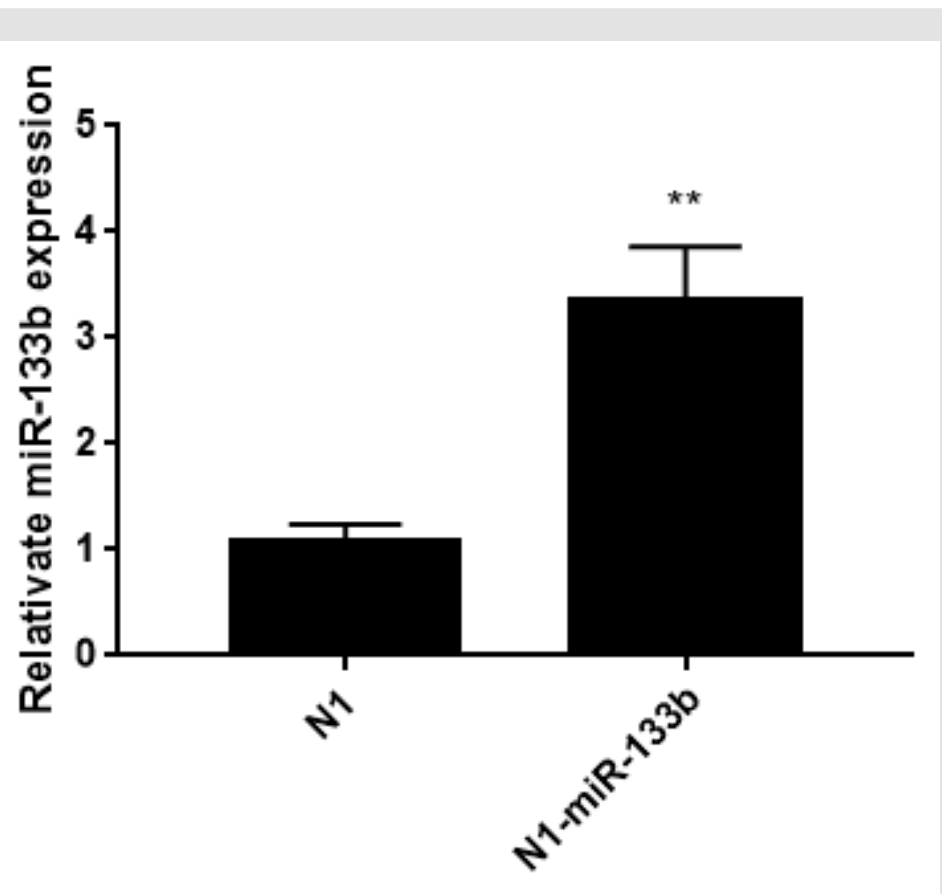

Figure 7: Relative miR-133b expression in $3 \mathrm{~T} 3$ cells after transfecting $48 \mathrm{~h}$.

Note: Date represent means $\pm S D ;{ }^{* *}$ represented extremely significant difference $(p<0.01)$

\section{Discussion}

MiRNA can regulate a large number of target genes independently by not completely complementary with specific mRNA, and participate in different cell signaling and metabolic pathways, as well as physiological and biochemical pathways such as cell proliferation, differentiation and apoptosis [10]. The development of animal muscle tissue is affected by a variety of regulatory factors, including myogenic regulatory factors MyoD, Myf5, MyoG;Pairing box factors Pax3, Pax7, etc. In recent years, more and more people pay attention to the research of noncoding RNA in muscle development, including Long non-coding RNA(lncRNA), circular RNA (circRNA) and miRNA [11]. MiRNA mainly inhibits its translation by binding the seed sequence (2-8 nucleotides from the 5 'end) to the target mRNA. MiR-1, miR-133, mir-486 and miR-206 are collectively referred to as muscle specific miRNAs, which are highly conserved in muscle tissues of different species [12]. Mir-486 participates in IGF-1 / Akt / mTOR signaling pathway to regulate skeletal muscle cell size, and also acts as a signal molecule of myogenic inhibitor [13]. After knocking out miR206 in C2C12 cells by CRISP-CAS9 technology, it was found that muscle development was slow, and ADAM19, BGN, Cbx5, Smarce1, and Spg20 were identified as its direct targets [14]. MiR-1 can promote muscle development by inhibiting the post transcriptional expression of HDAC4 in goats [15].

This study found that miR-133b plays an important role in muscle development. In disease treatment, miR-133b can reduce the harmful effects of Duchenne muscular dystrophy [16]. It can alleviate atherosclerosis by regulating vascular smooth muscle [17]. In livestock production, miR-133b can regulate the proliferation of bovine skeletal muscle satellite cells by targeting SP1 gene [18], and together with miR-1a and miR-206a to regulate muscle development of Tongcheng pig and landrace pig [19]. In this study, we constructed the tissue expression profile of Duroc Landrace pigs, and found that miR-133b was the highest expressed in dorsal muscle and myocardium, indicating that miR-133b may be related to muscle development. By constructing miR-133b overexpression vector, qPCR detection showed that the expression level of pegfpn1-mir-133b was significantly higher than that of the control group, indicating that miR-133b has a certain role in animal muscle development. These results indicate that miR-133b is expressed in 3T3 cells and specifically expressed in different tissues of Duroc Landrace pigs. This study provides a theoretical basis for further exploring the mechanism of miRNA in pigs and opens up a new way to improve the quantity and variety of pork.

\section{Conflict of Interests}

None.

\section{References}

1. Dong P, Mai Y, Zhang Z, Mi L, Wu G, et al. (2014) MiR-15a/b promotes adipogenesis in porcine pre-adipocyte via repressing Fox01. Acta Biochimica Et Biophysica Sinica 46(7): 565-571.

2. Kloosterman WP, Plasterk RHA (2006) The Diverse Functions of MicroRNAs in Animal Development and Disease. Developmental Cell 11(4): 441-450. 
3. Chen W (2014) Sequencing and characterization of miRNA and mRNA transcriptome of longissimus dorsi muscle in Laiwu and white pigs. Dr. Uncarried, Shandong Agricultural University.

4. Wang Wei, Guan Shuangbao, Wang Pengfei, Huang Xiaoyu, Xie Huihui, et al. (2020) Expression of miR-204 in porcine tissues and screening of important target genes.Acta Agriculturae Zhejiangensis 32(09): 15641573.

5. Li X (2020) MiR-208b regulates skeletal muscle fiber type transformation by inhibiting metTL8 expression.

6. Nohata N, Hanazawa T, Enokida H, Seki N (2012) microRNA-1/133a and microRNA-206/133b clusters: Dysregulation and functional roles in human cancers. Oncotarget $3(1)$.

7. Guo YY (2020) Expression level and significance of miR-133b in type 2 diabetic nephropathy. Uncarried Master, Hunan Normal University.

8. Zhou XH, Li YM, Li S, Teng XL (2019) Expression and prognostic value of miRNA-133b in gastric cancer. Cancer Progress 17(18): 2186-2191.

9. Rotelli MT, Refolo MG, Lippolis C, Cavallini A, Picciariello A, et al. (2020) The role of miRNA-133b and its target gene SIRT1 in FAP-derived desmoid tumor. Oncotarget 11(26).

10. Iorio MV, Croce CM (2012) Causes and Consequences of MicroRNA Dysregulation. The Cancer Journal 18(3): 215-222.

11. Bai Fengting, Li Lin, Chen Junhao, Zhao Bei, Peng Xinyu, et al. (2020) Research progress of non-coding RNA and skeletal muscle development. Chinese Journal of Animal Science and Veterinary Medicine 47(11): 3584-3594.

\section{ISSN: 2574-1241}

DOI: 10.26717/BJSTR.2021.38.006089

Mingsheng Jiang. Biomed J Sci \& Tech Res

(C) (P) This work is licensed under Creative Commons Attribution 4.0 License

Submission Link: https://biomedres.us/submit-manuscript.php
12. Townley-Tilson WHD, Callis TE, Wang DZ (2010) MicroRNAs 1, 133 and 206: critical factors of skeletal and cardiac muscle development, function, and disease. Int J Biochem Cell Biol 42(8): 1252-1255.

13. Hitachi K, Nakatani M, Tsuchida K (2014) Myostatin signaling regulates Akt activity via the regulation of miR-486 expression. International Journal of Biochemistry and Cell Biology 47.

14. Salant Tat, Goodrich, Kugel (2020) miR-206 knockout shows it is critical for myogenesis and directly regulates newly identified target mRNAs. RNA Biology 17(7)

15. Sui M, Zheng Q, Wu H, Zhu L, Ling Y, et al. (2020) The expression and regulation of miR-1 in goat skeletal muscle and satellite cell during muscle growth and development. Animal Biotechnology 31(5).

16. Taetzsch T, Shapiro D, Eldosougi R, Myers T, Settlage RE, et al. (2021) The microRNA miR-133b functions to slow Duchenne muscular dystrophy pathogenesis. The Physiological Society 1(599): 171-192.

17. Huadong L, Wei X, Feng L, Feng L, Junbo H, et al. (2019) MicroRNA-133b regulates the growth and migration of vascular smooth muscle cells by targeting matrix metallopeptidase 9. Pathology, research and practice 215(5).

18. Wang YH (2014) Effects of miR-133b on proliferation of bovine skeletal muscle satellite cells.

Uncarried Master, Northeast Agricultural University.

19. (2011) Master, Unpublished, Chinese Academy of Agricultural Sciences (2011) Expression analysis of microRNA-1A, 133B, and 206A in porcine muscle growth. Uncarried Master, Hunan Agricultural University.

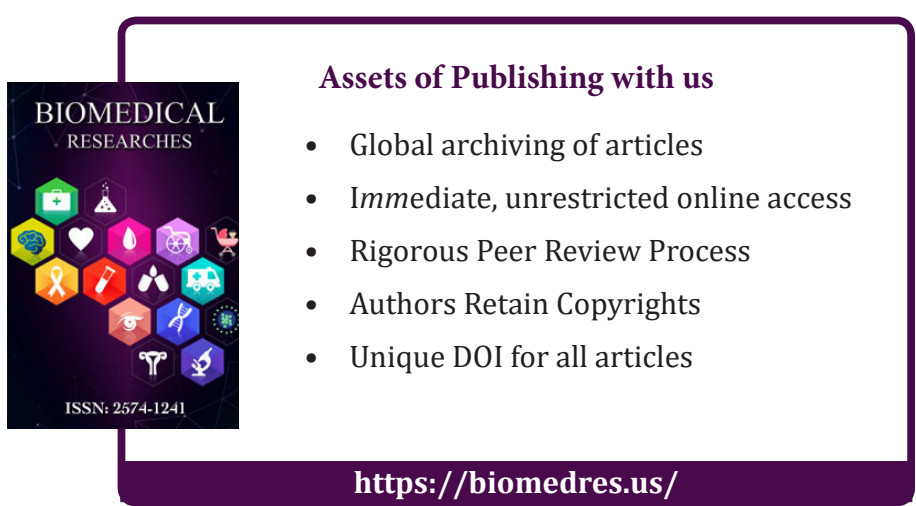

